\title{
Clinical Behavior of Dengue and Chikungunya Infections in the Instituto Mexicano del Seguro Social
}

\author{
Joel Navarrete-Espinosa1, Joaquín Gonzalez-Ibarra², Clara E. Santa Cruz-Tinoco², \\ Cesar R. Gonzalez-Bonilla², Miguel Pacheco-Hernández², José E. Muñoz-Medina² \\ ${ }^{1}$ División de Enfermedades Transmisibles, Coordinación de Vigilancia Epidemiológica, IMSS, México City, \\ México \\ ${ }^{2}$ División de Laboratorios de Vigilancia e Investigación Epidemiológica, Coordinación de Vigilancia \\ Epidemiológica, IMSS, México City, México \\ Email: joel.navarrete@imss.gob.mx
}

Received 5 January 2016; accepted 18 March 2016; published 21 March 2016

Copyright (C) 2016 by authors and Scientific Research Publishing Inc. This work is licensed under the Creative Commons Attribution International License (CC BY). http://creativecommons.org/licenses/by/4.0/

(c) (i) Open Access

\begin{abstract}
Arbovirus infections have increased worldwide in the last years and specific differential diagnosis may be difficult owing to their similar clinical presentations. Here we describe and compare the clinical manifestations of dengue and chikungunya fever. Material and Methods: In order to portray the clinical behavior of both diseases, a cross-sectional study was performed including patients insured by the Mexican Institute for Social Security (IMSS), their demographic data, and clinical signs and symptoms. Laboratory parameters were analyzed. Results: One thousand four hundred and ninety-five dengue and 1810 chikungunya cases were studied. All patients presented fever, whereas headache, myalgias, mild arthralgias, and ocular pain were more frequent in dengue cases. Severe incapacitating polyarthralgias, arthritis, and conjunctivitis were present only in chikungunya cases. Also, edema and exanthema were more frequent in patients with chikungunya. Liquid leak to tissues and hemorrhage were more frequent among dengue cases. Platelet counts were lower in hemorrhagic dengue $(P<\mathbf{0 . 0 0 1})$. Laboratory parameters were altered between the second and third day of evolution and tended to return to normal from the fifth day. Discussion: The clinical features observed in this group of patients may contribute to the differential diagnosis of both diseases. The cardinal signs of severity were not present in all cases of any of the two diseases. It is important to consider these issues in order to establish an early diagnosis and improve the prognosis of patients.
\end{abstract}

\section{Keywords}

Chikungunya, Behavior, Clinical

How to cite this paper: Navarrete-Espinosa, J., et al. (2016) Clinical Behavior of Dengue and Chikungunya Infections in the Instituto Mexicano del Seguro Social. Open Journal of Medical Microbiology, 6, 23-31. 


\section{Introduction}

Over the last few decades, climatic changes caused by global warming, deforestation, and invasion of wild habitats may have aided the appearance, reemergence, and spread of many infectious diseases, such as Ebola, cholera, leptospirosis, as well as those transmitted by vectors, and specially dengue, encephalitis by Niles virus, malaria, chikungunya fever, and more recently Zika fever, among others [1]-[3]. Other factors, such as the speed in communications and transportation of merchandise and persons, as well as social, economic, cultural, and traditional factors can be added to the list, and are considered as major causes for the introduction of these diseases [4].

This situation has been particularly evident on the American continent, where currently there are major outbreaks of different vector-transmitted diseases, specifically by Aedes aegypti. Dengue and chikungunya virus (CHIKV) are the most relevant at present.

The first large epidemic of dengue on the American continent occurred in the eighteenth century. From there, it affected almost all the countries in the region, especially in Latin America and the Caribbean. Although hemorrhagic dengue (HD) was described in Southeast Asia in 1960, it is suspected that it first spread through Greece with a high mortality rate observed in the early twentieth century [5] [6]. The first large outbreak of HD in Cuba occurred during 1981, with a consequent high economic and social burden for the country.

Since that date, the number of countries affected has increased as a consequence of the spread of the vector and the circulation of the four serotypes of the virus that produce the infection virtually throughout the continent, to the extent that it has become a true problem for public health [7]-[9].

Currently, there are more than 18 countries in Latin America and the Caribbean where the four serotypes are reported to cause dengue. By week 36 of 2015, more than 1.5 million probable cases were reported, and 25\% of them were confirmed by laboratory studies. Brazil, Mexico, and Colombia accounted for $86 \%$ of the notified cases. Severe cases represent only $0.3 \%$ of the total cases, with a mortality rate of $14 \%$; a number that exceeds the satisfactory standard considered by the World Health Organization [10] [11] if adequate management of the severe cases was to be achieved. The figures demonstrate that dengue is a very serious public health problem in the countries mentioned.

In the same way, the evidence of infection by the CHIKV goes back to the eighteenth century; however, the virus was not recognized until the second half of the past century, when there was an outbreak in Tanzania. Since then, the expansion of the disease has been constant, affecting many countries of Africa, Asia, and islands in the Indian and Pacific Oceans. During the present century, the disease has reached European countries (Italy) and the Caribbean. Currently, the disease has arrived in the American continent and has spread to all those countries where Aedes aegypti is found [12].

Up to week 40 of the 2015, 20 countries in Latin America and the Caribbean notified more than 0.5 million cases, of which $4 \%$ have been confirmed. In spite of this, the disease is described as non-fatal, with 62 deaths having been reported to date, corresponding to a mortality rate of $0.01 \%$. The countries reporting the highest number of cases are Colombia, Honduras, Nicaragua, El Salvador, and Ecuador, which combined account for 91\% of total notifications [13]. In Mexico, despite the fact that transmission has been high, only cases confirmed by laboratory studies are reported (7942). Nevertheless, the transmission of this infection started in the American continent during 2014, and the recorded numbers show that the disease has become a public health problem nationally and internationally.

Although both diseases are classified in the group of arboviruses (arthropod-borne), dengue is produced by a flavivirus (Flaviviridae group), and it is classified within the syndromes that produce hemorrhagic fevers. On the other hand, the CHIKV is an alphavirus (Togaviridae group) and the illness it produces is classified within the syndromes that cause arthritis and exanthema [14]. However, the initial symptoms of both diseases are very similar [12] [15]-[18].

In countries like Mexico, these facts have caused a problem for clinicians when establishing the diagnosis, for although the diseases are all viral, the treatment of each one is different and involves a risk when giving treatment and establishing a prognosis, especially in sick persons who are at extremes of life, or those with comorbidities.

The present work has been conducted for this reason, and to know the main clinical characteristics of both diseases. The aim is to develop tools that will help the clinician to differentiate both diseases and thus decrease the risk of complications or death in individuals affected by these infections. 


\section{Material and Methods}

A transversal study was performed including laboratory-confirmed cases notified by epidemiologists in the institutional surveillance systems for dengue and chikungunya from 2013 to 2015. From the database, non-fatal cases were included, with positive diagnostic tests according the specific normativity for each disease [19] [20]. According to these criteria, at first contact with the patient and with those with an evolution of less than 5 days, $3 \mathrm{~mL}$ blood samples were drawn, conserved in a cold net at $-8^{\circ} \mathrm{C}$ to $4^{\circ} \mathrm{C}$, and sent to the Epidemiology Central Laboratory at the Centro Médico "La Raza” (IMSS), where they were processed by RT-qPCR for each disease, as reported elsewhere [19]-[21]. Dengue was confirmed by NS1 antigen-capture ELISA (Dengue Early ELISA, Panbio Cat. E-DEN02P) when the sample was collected in the first 5 days of the clinical symptoms or by IgG/IgM ELISA after that period (Panbio, Dengue IgM capture ELISA Cat. E-DEN01M, Dengue IgG capture ELISA Cat. E-DEN02G).

Personal information for each patient and corresponding clinical data were obtained from the epidemiologic studies and from the specific information databases. Age, sex, and main signs and symptoms of the cases were recorded, as well as platelet and hematocrit values.

Dengue cases were classified in two groups according to the clinical evolution and its severity: dengue fever (DF) and hemorrhagic dengue fever (HDF) (CIE 2015). The first group included the non-severe dengue cases and dengue cases with signs of alarm (DFSA) and the second included the severe dengue cases. The database does not identify severe or chronic cases of chikungunya which tend to develop arthritis.

A new database was prepared with the obtained information, and simple frequencies, percentages, and central tendency measurements were obtained; the main signs and symptoms of each disease were compared, as well as the laboratory parameters between the two groups. All analyses were performed using Stata ${ }^{\mathrm{TM}} 10.0$ (Stata Corp, College Station, Tex.). The mean values of platelets and hematocrit in both groups were compared with Bartlett's test for equal variances.

\section{Results}

In total, 1495 dengue cases were studied and confirmed by RT-qPCR. They were notified by 21 delegations from the Mexican Institute for Social Security (IMSS) during 2013 (Figure 1), and no identified infections by the CHIKV in the country were identified during the period concerned. From these, serotype 1 was identified in 820 cases, serotype 2 in 615, serotype 3 in 2, and serotype 4 in 58 cases. In the same way, the diagnosis of DF was established in 837 cases, DFSA in 378, and HDF in 280. At the time, and for the purposes of this analysis, the cases were grouped into DF (1215 cases) and HDF (280 cases). From the total number of cases, 51\% were males and 49\% were females. The highest proportion of cases corresponded to the age group of 25 to 44 years (37\%), followed by those of 15 to 24 years (22\%), 5 to 14 years (18\%), 45 to 64 years (15\%), 65 years old or more (4\%), and 1 to 4 years (4\%).

Similarly, 1810 cases of chikungunya were confirmed by RT-qPCR studies, notified by 23 delegations of the IMSS during 2015 (Figure 2), the year in which the transmission of the infection started in the country. A total of $62 \%$ of cases corresponded to females and $38 \%$ to males. The highest proportion of patients was within the age group of 25 to 44 years (40\%), followed by those of 45 to 64 years (23\%), 15 to 24 years (14\%), 5 to 14 years (11\%), 65 or more (8\%), and finally 4\% who were 4 years old or less.

According to the clinical behavior of DF, HDF, and chikungunya cases, fever was present in all the patients. The presence of headache, myalgia, mild arthralgia, and ocular pain were more frequent in dengue cases, especially in those classified as DF (Table 1).

Severe polyathralgia (incapacitating), arthritis, and conjunctivitis were only present in the chikungunya cases; and exanthema was also more frequent in the latter. Also, edema was more frequent in the cases of chikungunya, especially in the joints.

In contrast, the leak of liquids characterized by petechiae, ecchymosis, bruising, ascites, pleural effusion, and mottled skin were more common in cases of dengue; specifically the most serious (HDF). Hemorrhages of different localization were also more common in severe cases of dengue.

In this sense, although hemorrhagic manifestations only described only for rare forms of infection by the CHIKV, they were also observed in these cases (Table 1).

With respect to the laboratory parameters, the average of the platelet values in the chikungunya cases was $221,991$ ( $\pm 60,614), 168,150$ ( \pm 49,017) for DF, and 65,367 ( \pm 34,394) for HDF. The difference was significant 


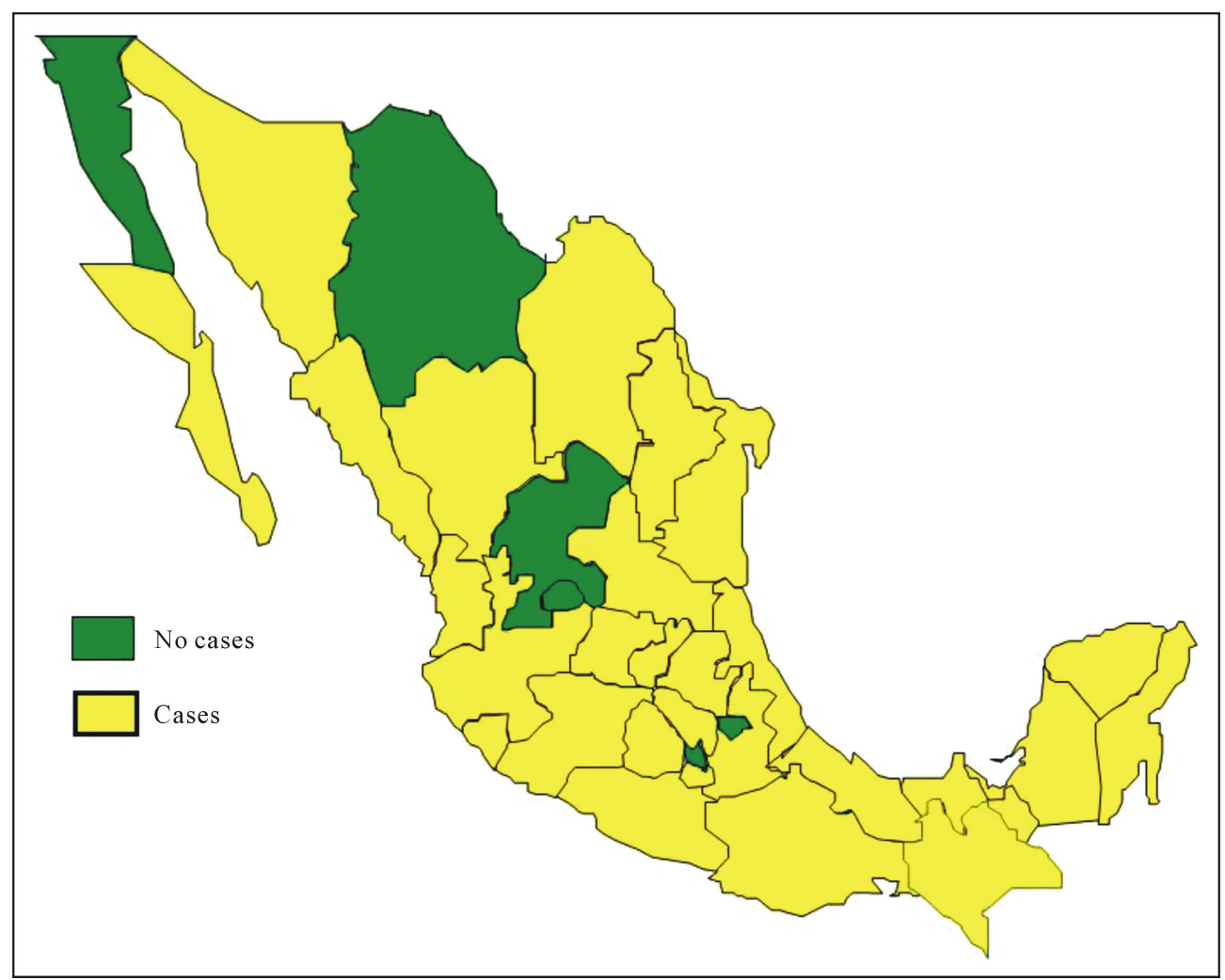

Figure 1. Delegations with Dengue cases reported by the Mexican Institute for Social Security, 2015.

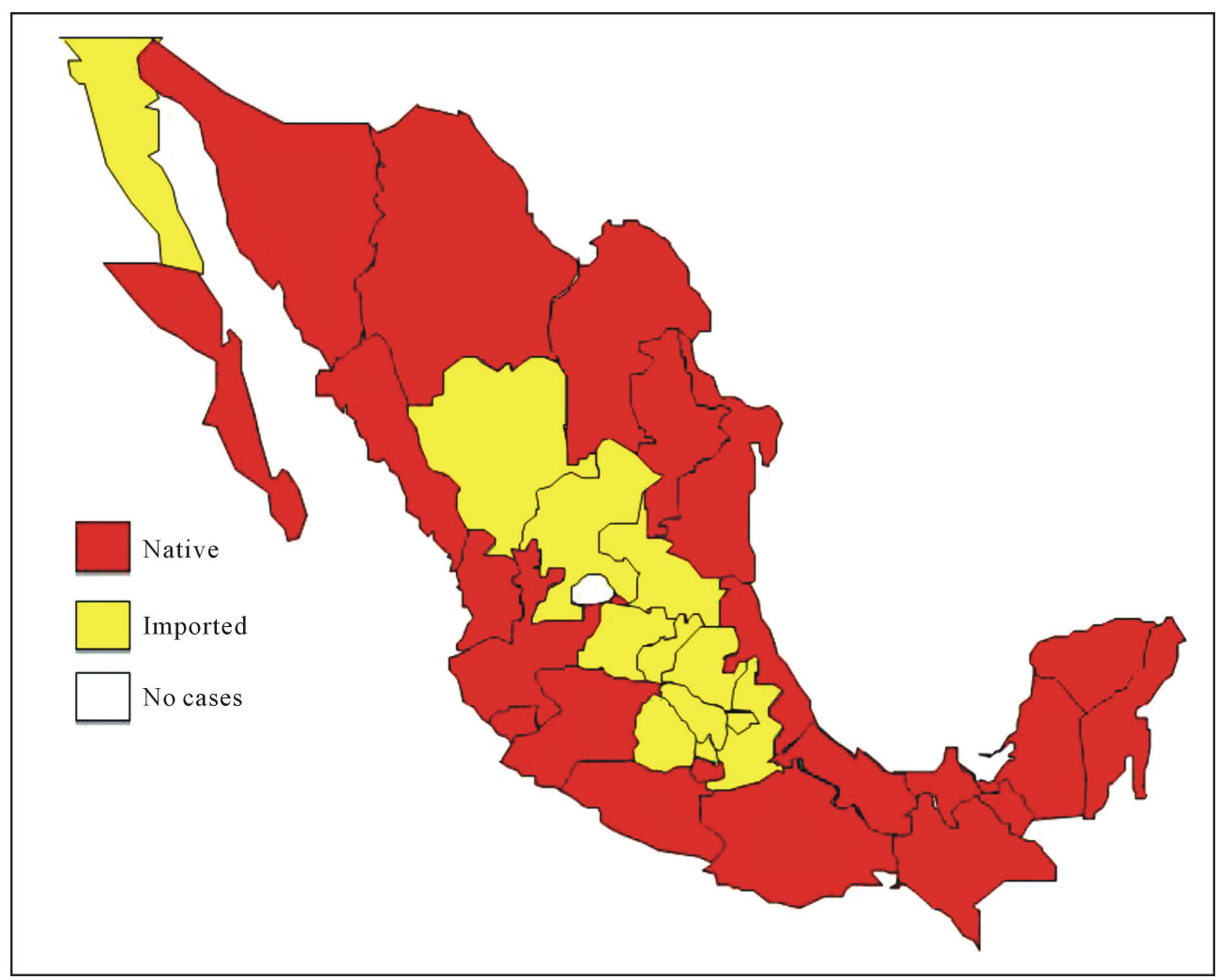

Figure 2. Delegations with Chikungunya reported by the Mexican Institute for Social Security, 2015. 
Table 1. Main signs and symptoms in the cases.

\begin{tabular}{|c|c|c|c|}
\hline Clinical Symptoms & $\begin{array}{c}\text { Chikungunya } \\
\text { (n=1812) } \\
\%\end{array}$ & $\begin{array}{c}\text { Dengue Fever } \\
(\mathrm{n}=1215) \\
\%\end{array}$ & $\begin{array}{c}\text { Hemorrhagic } \\
\text { Dengue Fever } \\
(\mathrm{n}=280) \\
\%\end{array}$ \\
\hline Fever & 100 & 100 & 100 \\
\hline Headaches & 94 & 97 & 94 \\
\hline Myalgia & 94 & 95 & 91 \\
\hline Arthralgias & 80 & 93 & 90 \\
\hline Polyarthralgias-Incapacitating & 84 & 0 & 0 \\
\hline Ocular Pain & 59 & 83 & 73 \\
\hline Exanthema & 66 & 25 & 37 \\
\hline Arthritis & 64 & 0 & 0 \\
\hline Conjunctivitis & 19 & 0 & 0 \\
\hline Liquid Leak & 9 & 5 & 56 \\
\hline Petequiae & 4 & 4 & 45 \\
\hline Ecchymosis & 0.2 & 0 & 5 \\
\hline Hematomas & 0.3 & 0 & 4 \\
\hline Positive Tourniquet & 0 & 1 & 14 \\
\hline Ascites & 0.1 & 0 & 3 \\
\hline Edema & 12 & 0 & 3 \\
\hline Pleural Effusion & 0 & 0 & 2 \\
\hline Mottled Skin & 0.3 & 0 & 5 \\
\hline Hemorrhage & 1.3 & 4 & 32 \\
\hline Gingival & 1 & 1 & 17 \\
\hline Epistaxis & 1 & 2 & 9 \\
\hline Hematemesis & 0.3 & 0 & 4 \\
\hline Melena & 0.2 & 0 & 3 \\
\hline
\end{tabular}

for the latter ( $F=1225.5, P=0.001)$. The behavior of the platelets according to the days of evolution in the groups are shown in Figure 3 ( 1 = Chikungunya, $2=$ DF and $3=\mathrm{HDF})$.

With regard to hematocrit, the averages were $39.4( \pm 4.7)$ for the chikungunya cases, $40.5( \pm 4.7)$ for DF, and 41.9 ( \pm 5.7 ) for HDF. The comparison among the groups was significant for the last group $(F=34.6, P=0.001)$. Nevertheless, the behavior of the hematocrit values according to the number of days of evolution in the patients did not show significant differences (Figure 4; 1 = Chikungunya, $2=$ DF and $3=$ HDF).

Finally, the count of leukocytes was only performed in the chikungunya cases and the average value of these was 5947 ( \pm 2583 ), with a minimum value of 2000 and a maximum of 39,700. According to the days of evolution, the clinical data of the cases showed that the leukocyte values decreased in the second day, and after the sixth day they presented recuperation towards normal values (Figure 5). 


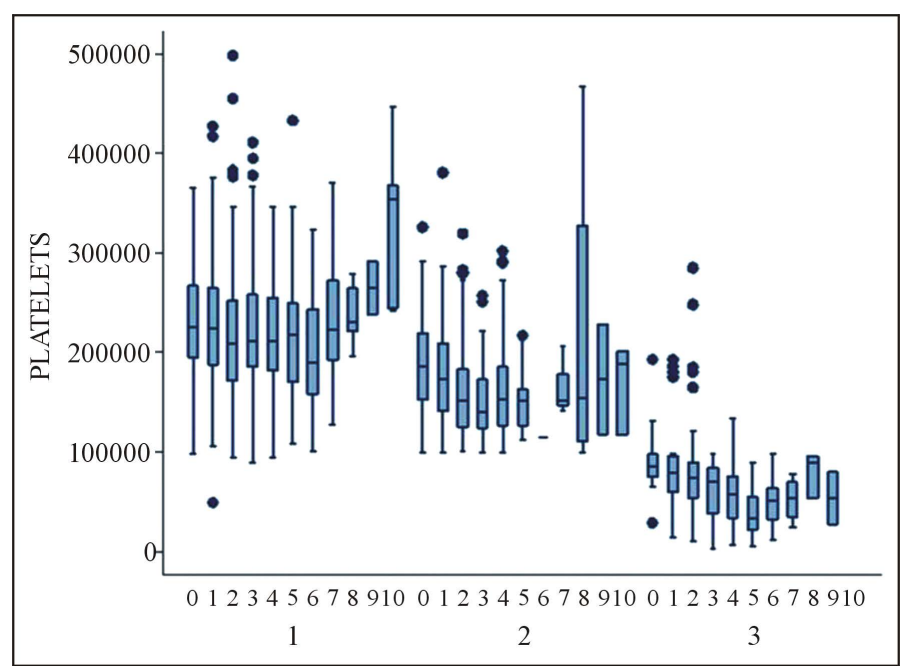

Figure 3. Behavior of platelets in the cases according to the days of evolution.

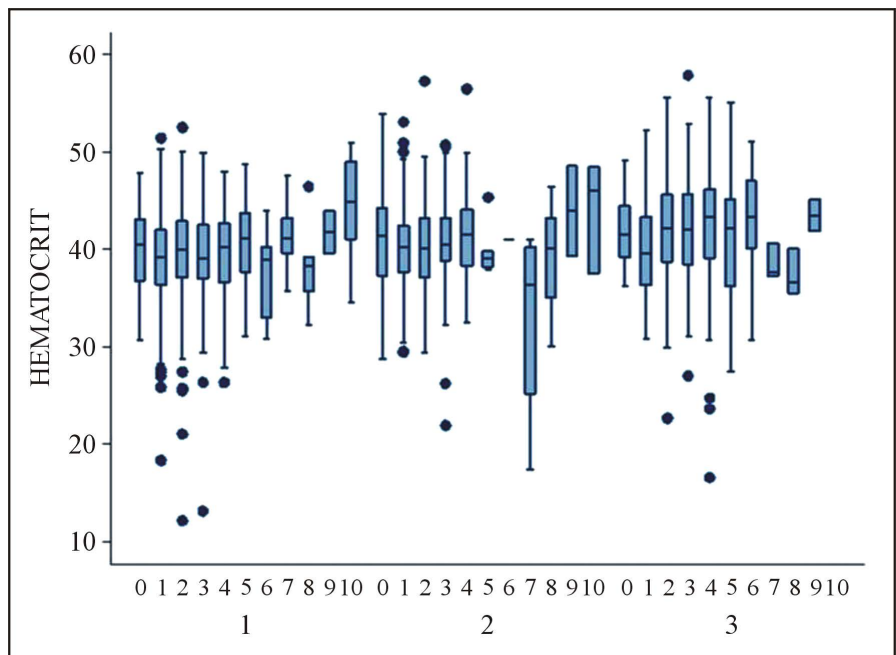

Figure 4. Behavior of Hematocrit in the cases according to the days of evolution.

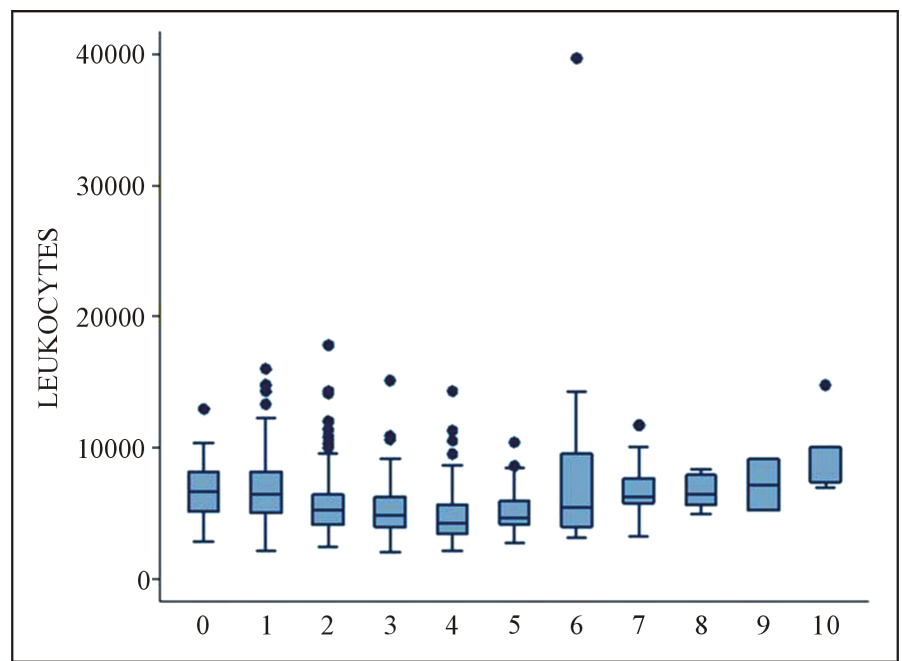

Figure 5. Leukocyte behavior in Chikungunya cases according to the days of evolution. 


\section{Discussion}

Diseases transmitted by vectors currently constitute a worldwide threat; they have never represented a problem of the magnitude that they do today. Some of the most important among these are malaria and yellow fever, which are known to have caused havoc among humans for many centuries. Similarly and more recently, dengue and leishmaniosis have affected large populations, mainly in tropical regions. Currently, deforestation and global warming have favored changes that together with the invasion by humans of different wild habitats have exposed people to other diseases, such as Nile encephalitis and infections by the chikungunya and Zika viruses, which only affected some groups of animals in their wild environment in the past.

The evolution and adaptation of the different vectors have played an important role in the spreading of these diseases. Such is the case of Aedes aegypti, which has currently spread throughout the world, and in almost all of the American continent; it not only proliferates in tropical weather, as was considered to be the case for some time, but its capacity of adaptation has allowed it to invade subtropical climates, and even those where it was thought its proliferation was impossible [22].

This fact is relevant because the vector is capable of transmitting different viral diseases, such as yellow fever, dengue, Mayaro, and chikungunya fever, and recently, infection by the Zika virus has been observed [23]. For this, the American continent is currently registering the worst pandemic produced by the spread of this vector. The climatic changes, as well as the low success of the eradication campaigns, have permitted high densities of this vector, and thus the intense transmission of these diseases. The problem is even greater if we consider that Aedes aegypti can transmit two or more of these infections simultaneously.

In this sense, most of the countries in Latin America and the Caribbean register cases of dengue, and Brazil, Mexico, and Colombia, report hyperendemicity, with a large number of severe cases and a high demand for medical attention; in addition, an important underregistration of the infection is suspected, which demonstrates that dengue is a true problem in these countries [10].

Due to its rapid spread, this same situation is observed regarding the infection by the CHIKV, because in less than two years after its presence was detected in America, it has demonstrated an autochthonous transmission in at least 15 countries of the continent, with an overflowing number of cases.

These facts have led to consequent difficulties for the estimation of the true magnitude of the problem, because at the beginning of the infection, the clinical symptoms of both are very similar and have few differences, making the diagnosis and the real estimation of the incidence of each disease more difficult. This is evident if we consider the positivity index of the diagnostic tests (RT-qPCR), which in Mexico is 31\% for DF cases and $62 \%$ for HDF, as well as 59\% for chikungunya cases, which indicates that at least half of the cases notified as dengue could represent the occurrence of other infections, such as rickettsiosis and leptospirosis [24], or in the case of chikungunya it could be the presence of the Mayaro virus, which shares phylogenetic (alphavirus) and clinical characteristics with chikungunya [25] that have been overlooked in our country to date [26].

On the other hand, regarding the clinical behavior, dengue is characterized by the presence of fever, headache, myalgia, arthralgia of variable intensity, and exanthema; this is accompanied by general malaise and other signs and symptoms that may or may not be present [15]-[17]. Characteristically, mild-intensity presentations are indistinguishable in many infectious diseases, and only severe cases of dengue may manifest differently through hemorrhages, capillary fragility data, or leak of liquids. The infection by CHIKV also has an onset that may not be differentiated and simulate different infections; only severe cases, or very characteristic ones, can be diagnosed with certainty through the presence of incapacitating arthritis and edema in the joints [12] [18].

Nonetheless, according to the physiopathology of these diseases, the behavior of some laboratory parameters, such as platelets, may serve as a guide to differentiate cases of dengue and chikungunya. As was observed in this study, the values of this component are lower and show a decreased tendency in cases of dengue, and especially in severe cases of HDF, in contrast to infection by the chikungunya virus, in which thrombocytopenia is absent or determined by other factors of comorbidity. Likewise, as expected, the hematocrit behavior shows differences in both diseases, with a higher concentration in dengue cases.

For both parameters, the third day of evolution of the infection is seen as a critical point, when a decrease of platelets and an increase in the hematocrit are observed up to the sixth or seventh day, when a recuperation of values towards normality is observed (Figure 3, Figure 4). This phenomenon is also observed in the behavior of leukocytes (Figure 5). In relation to this last parameter, and although literature refers to a decrease in the count of leukocytes during the infection, it calls our attention to the fact that in this series of cases they are elevated in 
elder patients, and with a marked clinical presentation, which could have a relation with the presence of comorbidity or the presence of concomitant infections. According to the previous, being unable to study the clinical presence and behavior of the cases with simultaneous infections of dengue and chikungunya or any of these cases with other infections was a limitation in this study.

Nevertheless, the clinical behavior presented in the studied cases shows correlation with literature and may be used as a base for the diagnosis of both diseases, considering the cardinal signs and symptoms of each disease, especially in those cases in which they have a characteristic behavior. However, it is worthy of mention that according to the observations in this study, the cardinal signs in severe dengue cases (leak of liquids and hemorrhages) and chikungunya (incapacitating polyarthralgias and edema in joints) were not present in all the patients (Table 1).

This aspect is important and it has substantial implications for the diagnosis and initial treatment of the patients. For this reason, the availability of specific tests that allow a timely diagnosis to decrease complications and improve the prognosis of the patients is important. Furthermore, effective epidemiologic surveillance programs and subsequent estimates of the real occurrence of all these diseases would justify implementing activities to control the vectors.

Therefore, if it is true that infections transmitted by a vector are a real problem in public health worldwide, especially on the American continent, it is also true that by selecting the best, high-impact, ample-coverage antivector strategies permanently, their transmission could be minimized. Basically, the development of social and sanitary infrastructure is required to make water and drainage available to all houses, as well as the collection and correct deposition of garbage. Health education, including high-impact media penetration, is necessary to encourage and favor social development in vulnerable populations. Other mechanisms, such as the indiscriminate use of pesticides and vaccines, although helpful, may have consequences for the environment, transform the risk groups, and delay the problem.

\section{Conclusions}

Arboviral infections are an increasing public health problem worldwide. The main diseases, dengue, and chikungunya fever, share clinical manifestations which present difficult and differential diagnoses, particularly in the first week of the disease. The cross-sectional study described here included the laboratory-confirmed cases reported by the IMSS surveillance system from 2013 to 2015, which has a national scope. In order to register a case, the operational definition of the survey system requires fever and one or more of the following symptoms: headache, myalgias, arthralgias, exanthema, or ocular pain. This could be a limitation in this study. As expected, severe incapacitating polyarthralgias and arthritis were present only in chikungunya cases, whereas hemorrhage was more frequent in dengue cases. The main difference in the laboratory parameters was observed in platelets, which were lower in HDF.

Control of these diseases will need the commitment of the national, state, and municipal authorities, as well as from the mass communication media and society in general to improve the environment and educate the community of the importance of decreasing potential breeding sites, establishing a permanent control of vector densities, and avoiding contact, and ultimately diminishing the transmission of these infections.

\section{References}

[1] Shope, R. (1991) Global Climate Change and Infectious Diseases. Environmental Health Perspectives, 96, $171-174$. http://dx.doi.org/10.1289/ehp.9196171

[2] Guzmán, M.G., Kourí, G. and Pelegrino, J.L. (2001) Enfermedades Virales Emergentes. Revista Cubana de Medicina Tropical, 53, 5-15.

[3] Kilbourne, E.D. (1990) New Viral Diseases. A Real and Potential Problem without Boundaries. JAMA, 264, 68-70. http://dx.doi.org/10.1001/jama.1990.03450010072033

[4] Randolph, S.E. and Rogers, D.J. (2010) The Arrival, Establishment and Spread of Exotic Diseases: Patterns and Predictions. Nature Reviews Microbiology, 8, 361-371. http://dx.doi.org/10.1038/nrmicro2336

[5] Monografía sobre la epidemiología del Dengue. Secretaría de Salud. Dirección General de Epidemiología. 1992.

[6] Kourí, G. (2006) El dengue, un problema creciente de salud en las Américas. Revista Panamericana de Salud Pública, 19, 143-145. http://dx.doi.org/10.1590/S1020-49892006000300001

[7] Jetten, T.H. and Focks, D.A. (1997) Potential Changes in the Distribution of Dengue Transmission under Climate 
Warming. American Journal of Tropical Medicine and Hygiene, 57, 285-297.

[8] Guzman, M.G. and Kourí, G. (2003) Dengue and Dengue Hemorrhagic Fever in the Americas: Lessons and Challenges. Journal of Clinical Virology, 27, 1-13. http://dx.doi.org/10.1016/S1386-6532(03)00010-6

[9] Gibbons, R.V. and Vaughn, D.W. (2002) Dengue: An Escalating Problem. BMJ, 324, 1563-1566. http://dx.doi.org/10.1136/bmj.324.7353.1563

[10] Number of Reported Cases of Dengue and Severe Dengue (SD) in the Americas. Boletín de la Organización Panamericana de la Salud. Epidemiological Week 36 (Updated 25 September 2015).

[11] Organización Panamericana de la Salud. Organización Mundial de la Salud (OMS). Dengue. Guías para el diagnóstico, Tratamiento, Prevención y Control. La Paz. Bolivia. OPS/OMS, 2010.

[12] Organización Panamericana de la Salud. Preparación y respuesta ante la eventual introducción del virus chikungunya en las Américas. OPS, Washington DC, 2011.

[13] Número de casos reportados de chikungunya en países o territorios de las Américas. Boletín de la Organización Panamericana de la Salud. Semana Epidemiológica 40 (actualizada al 09 de octubre de 2015).

[14] Heyman, D.L., Ed. (2011) El control de las enfermedades transmisibles. 19th Edition, OPS, Washington DC, $186-200$.

[15] Ramos, C., García, H. and Villaseca, J.M. (1993) Fiebre hemorrágica y síndrome de choque por dengue. Salud Pública de México, 35, 39-55.

[16] Martinez Torres, E. (1995) Dengue y dengue hemorrágico: Aspectos clínicos. Salud Pública de México, 37, $29-44$.

[17] Navarrete-Espinosa, J., Gómez-Dantés, H., Celis-Quintal, J.G. and Vázquez-Martínez, J.L. (2005) Clinical Profile of Dengue Hemorrhagic Fever Cases in Mexico. Salud Pública de México, 47, 193-200. http://dx.doi.org/10.1590/S0036-36342005000300002

[18] Vijayakumar, K.P., Anish, T.S.N., George, B., Lawrence, T., Muthukkutty, S.C. and Ramachandran, R. (2011) Clinical Profile of Chikungunya Patients during the Epidemic of 2007 in Kerala, India. Journal of Global Infectious Diseases, 3 , 221-226. http://dx.doi.org/10.4103/0974-777X.83526

[19] Norma Oficial Mexicana NOM-032-SSA2-2010, Para la vigilancia epidemiológica, prevención y control de las enfermedades transmitidas por vector.

[20] Procedimiento para la Aplicación del Nuevo Algoritmo para Diagnóstico por Laboratorio de Fiebre por Dengue y Fiebre Hemorrágica por Dengue. Secretaría de Salud. CENAVECE InDRE-RNLSP, 2008.

[21] Lineamientos estandarizados para la vigilancia epidemiológica y diagnóstico por laboratorio de Fiebre Chikungunya. Dirección General de Epidemiología. Secretaría de Salud, 2014.

[22] Herrera-Basto, E., Prevots, D.R., Zárate, M.L., Silva, J.L. and Sepúlveda-Amor, J. (1992) First Reported Outbreak of Classical Dengue Fever at 1700 Meters above Sea Level in Guerrero State, Mexico: June 1988. American Journal of Tropical Medicine and Hygiene, 46, 649-653.

[23] Organización Panamericana de la Salud. OMS. Alerta Epidemiológica. Síndrome neurológico, anomalías congénitas e infección por virus Zika. Implicaciones para la salud pública en las Américas. 1 de diciembre de 2015. http://www.paho.org/hq/index.php?option=com_docman\&task=doc_view\&Itemid=270\&gid=32286\&lang=es

[24] Dircio, M.S.A., González, F.E., Verdalet, G.M.S., Soler, H.E., Rivas, S.B., Altuzar, A.V.M. and Navarrete, E.J. (2012) Leptospirosis Prevalence in Patients with Initial Diagnosis of Dengue. Journal of Tropical Medicine, 2012, Article ID: 519701. http://dx.doi.org/10.1155/2012/519701

[25] de Oliveira Mota, M.T., Ribeiro, M.R., Vedovello, D. and Nogueira, M.L. (2015) Mayaro Virus: A Neglected Arbovirus of the Americas. Future Virology, 10, 1109-1122. www.futuremedicine.com

[26] Navarrete, E.J. and Gómez, D.H. (2006) Arbovirus causales de fiebre hemorrágica en pacientes del Instituto Mexicano del Seguro Social. Revista Médica del Instituto Mexicano del Seguro Social, 44, 347-253. 